\title{
Genetic space of radial plant growth
}

\author{
Radial growth of plants is one of the most prominent processes for generating biomass and the long-term \\ sequestration of carbon dioxide on Earth. Now, the concerted action of a large set of transcription factors on the \\ regulation of the process has been investigated.
}

\section{Thomas Greb}

T: he transformation of solar energy, water and carbon dioxide into biomass is fundamental for life on Earth. Plants are the main executers of this transformation, and their stem cells are hot spots for the production of food and long-lasting biomaterials. The cambium is one group of these stem cells mediating radial shoot and root growth as well as wood formation in many species, with the remarkable exception of the grasses ${ }^{1,2}$. In comparison to other plant stem cell systems, investigation of the cambium lagged behind for quite some time due to the cumbersome genetic analysis of more woody species and the somewhat hidden nature of the cambium in genetic model systems. Now, publishing in Nature Plants, Zhang and colleagues ${ }^{3}$ report on an unprecedented genetic analysis exploring the genetic space of radial plant growth in Arabidopsis thaliana. They find that, by modulating the activity of multiple transcription factor genes, distinct aspects of radial growth such as cell proliferation or differentiation can be accessed separately and, in some cases, extreme phenotypic alterations can be achieved.

The authors start from a transcriptional profiling approach of root cells characterized by the expression of the ARABIDOPSIS RESPONSE REGULATOR 15 (ARR15) gene. $A R R 15$-positive cells hold procambium identity and, by isolating cells marked by an ARR15-GFP reporter, subsequent RNA profiling and comparison of obtained profiles with publically available datasets, the authors identified 41 transcription factors whose activities are associated with cambium cells. After analysing the expression patterns, the authors dived into the genetic analysis of 32 transcription factor genes for which cambium-associated activity could be confirmed. Next, alterations of cambium activity in plant lines overexpressing those factors allowed the authors to select 13 transcription factors for which they established a regulatory network with the internal wiring dynamics simulated in silico. Regulatory interactions within the network were determined based on short-term inductions of respective gene activities and their effects on the mRNA levels of other network members. Indeed, plants deficient for more central members of the network showed stronger defects in cambium proliferation than plants deficient for more peripheral members, underlining its predictive power.

In the most central and remarkable part of the study, the authors scored cambiumassociated traits, such as cell diameter, total cell number or vessel number, in around 100 genetic backgrounds carrying different mutant allele combinations. In addition, the analysis of water-transporting vessel elements was interesting because they are the dominant cell-type determining physiological properties of wood and store up a large part of cambium-derived biomass. Their genetic tour de force allowed the authors to reveal antagonistic, redundant and epistatic relationships among the analysed genes in the regulation of these specific traits. In particular, and similarly to previous reports $^{4,5}$, double mutants in the WUSCHEL RELATED HOMEOBOX 4 (WOX4) and BREVIPEDICELLUS ( $B P$, also known as KNAT1) genes encoding homeobox transcription factors displayed the strongest defect in cambium proliferation observed in this study, suggesting a concerted role of both factors in promoting stem cell activity. Interestingly, histological inspection and transcriptional profiling of wox4 and other mutants suggested that WOX4, in addition to promoting cell proliferation, fulfils a role in vessel expansion.

Confirming this role, an increase of WOX 4 activity not only enhanced the number of cambium cells but also the amount of wood produced. Combining enhanced WOX4 activity with increased activity of other cambium-related transcription factors, or with a deficiency in the PETAL LOSS (PTL) gene that encodes a MYB-like transcription factor, increased these effects. In particular, in a PTLdeficient background, WOX4 activation leads to an extreme increase in radial root growth and the ectopic formation of cambium domains. At the end, the authors classified $P T L$ as a negative regulator of radial plant growth.

Resources generated in this study are certainly essential for promoting the field by providing a wealth of genetic tools and data. This is especially the case because distinct aspects of radial plant growth, such as cell proliferation or the production of vessel elements, can be accessed separately by modulating the activity of different sets of transcription factors. However, because immediate effects of changes in these transcription factor genes are difficult to monitor, a cautious interpretation of phenotypic alterations and of gene function is fundamental. For example, the classification of $B P$ as a promoter of cambium proliferation in the current study but also as a factor promoting differentiation of wood cells in previous reports ${ }^{6}$ reflects the challenges to understand the mechanism of radial plant growth. The development of experimental systems allowing direct observation of cambium activity, similar to organoids in animal research ${ }^{7}$ or ex planta approaches, is therefore more than desirable.

\section{Thomas Greb (iD) \\ Centre for Organismal Studies, Heidelberg University, Heidelberg, Germany. \\ e-mail: thomas.greb@cos.uni-heidelberg.de}

Published online: 8 October 2019

https://doi.org/10.1038/s41477-019-0536-3

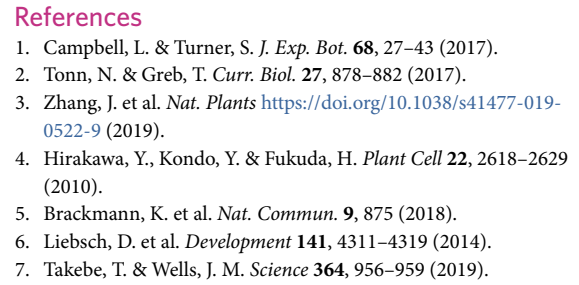

